\title{
Application of FCT in SPH Method
}

\author{
Xiaowang Sun ${ }^{1, a}$, Xiaojun Wang $^{1}$ and Jie Zhang $^{1}$ \\ ${ }^{1}$ University of Science and Technology of China, Hefei 230026, China; \\ ${ }^{a}$ xiaowang@mail.ustc.edu.cn
}

Keywords: FCT, SPH, stress wave.

\begin{abstract}
The computational scheme and the basic idea of the Flux-Corrected Transport (FCT) method are studied in the paper. With the numerical simulations of 1-D stress wave, its application to SPH are discussed and compared with SPH coupled with artificial viscosity. The result shows that FCT method is very efficient in eliminating unphysical oscillations behind the shock front and accurately describing shock waves.
\end{abstract}

\section{Introduction}

Up to now, the artificial viscosity ${ }^{[6]}$ is employed in $\mathrm{SPH}$ (smoothed particle hydrodynamics) method in order to eliminate the unphysical oscillations and Gibbs phenomenon behind the shock front. But the artificial viscosity artificially transforms kinetic energy into internal energy, not ensuring momentum conservation and energy conservation, so cannot obtain the actual physical picture. So its limitation in shock wave simulation is obvious.

FCT(Flux-Corrected Transport) method was created by Boris and Book ${ }^{[1-3]}$. They considered the FCT method as a scheme rather than a regular algorithm, which can be adopted in many high-order finite-difference methods. While original FCT method cannot completely eliminate oscillations behind wave caused by high-order dispersion. Zalesak ${ }^{[4]}$ developed FCT method by providing new limited form of anti-diffusion.

FCT method is used mostly in finite-difference method. As a Lagrange mesh-free method, SPH method is applied to large-deformation impact dynamics problem. Therefore, the combination of FCT and SPH has great practical value.

\section{SPH Method in Wave Simulation}

In the SPH method, the entire system is represented by a finite number of particles that carry individual mass and occupy individual space. The 1-D particle approximation for the spatial derivative used in this paper is

$$
\frac{\partial f}{\partial x}=\sum_{j}^{N} \frac{m_{j}}{\rho_{j}}\left(f_{j}-f_{i}\right) \frac{\partial W_{i j}}{\partial x}
$$

In Lagrange view, 1-D wave equation of continuous medium is written as

$$
\rho \frac{d u}{d t}=\frac{\partial \sigma^{x}}{\partial x}
$$

Employing equation (1), SPH approximation of the wave equation is obtained as:

$$
\frac{d u_{i}}{d t}=\frac{1}{\rho_{i}} \sum_{j=1}^{N} \frac{m_{j}}{\rho_{j}}\left(\sigma_{j}^{x}-\sigma_{i}^{x}\right) W_{i j, i}
$$

The artificial viscosity developed by Monaghan is omitted in this paper(see ref[6]).

\section{FCT Method}

For an arbitrary time-related function $V$, SPH equation in time direction can be written as,

$$
V_{i}^{n+1}=f\left(V_{i}^{n}, \Delta t, \Delta x\right)
$$


Boris and Book gave the general form of FCT method:

1. Transport calculation. The uncorrected $\tilde{V}_{i}^{n+1}$ is obtained by equation (4) .

$$
\tilde{V}_{i}^{n+1}=f\left(V_{i}^{n}, \Delta t, \Delta x\right)
$$

2. Diffusion flux of time $n$, where $\eta_{1}$ is diffusion coefficient.

$$
F_{i}^{n}=\eta_{1}\left(V_{i+1}^{n}-V_{i}^{n}\right)
$$

3. Diffusion calculation. Where small oscillations is eliminated.

$$
\bar{V}_{i}^{n+1}=\tilde{V}_{i}^{n+1}+F_{i}^{n}-F_{i-1}^{n}
$$

4. Anti-diffusion calculation, where $\eta_{2}$ is anti-diffusion coefficient.

$$
F_{i}^{n+1}=\eta_{2}\left(\tilde{V}_{i+1}^{n+1}-\tilde{V}_{i}^{n+1}\right)
$$

5. Limit-condition of anti-diffusion flux, where $\Delta_{i}=\bar{V}_{i+1}^{n+1}-\bar{V}_{i}^{n+1}, S=\operatorname{sign}\left(F_{i}^{n+1}\right)$.

$$
F_{i}^{c}=S \cdot \max \left[0, \min \left(S \cdot \Delta_{i-1}, S \cdot \Delta_{i+1},\left|F_{i}^{n+1}\right|\right)\right]
$$

6. Anti-diffusion. Where the curve is made sharper.

$$
V_{i}^{n+1}=\bar{V}_{i}^{n+1}-F_{i}^{c}+F_{i-1}^{c}
$$

FCT adds a diffusion item to dispersion flux format, eliminating numerical oscillations near shock wave front. And the anti-diffusion calculation compensates diffusion error, while causing oscillations. That's where the limit-condition of anti-diffusion flux arise, which can eliminate the new oscillations, and get the stable solution. FCT is applied in conservation form, conserving momentum and energy of the system.

Boris and Book's method cannot completely remove the high-order dispersion oscillation. Zalesak brought higher accuracy to FCT method by modifying it and giving a new limit-condition of anti-diffusion flux. Zalesak's method is tedious because it needs a low-order equation and a high-order equation.

In this paper, FCT method is modified on the base of Boris and Book's work, limit-region of anti-diffusion flux is magnified, that is proved effective. Limitation of anti-diffusion flux avoid new extremum. According to equation (9), extremum at $i$ point is avoided by getting the minimum value among $S \cdot \Delta_{i-1}, S \cdot \Delta_{i+1}$ and $\left|F_{i}^{n+1}\right|$. In actual computation, it is found that the limit-region is too small to produce little oscillation. So $S \cdot \Delta_{i-2}, S \cdot \Delta_{i}$ and $S \cdot \Delta_{i+2}$ are added into the limit-condition equation, and equation (9) evolves into:

$$
F_{i}^{c}=S \cdot \max \left[0, \min \left(S \cdot \Delta_{i-2}, S \cdot \Delta_{i-1}, S \cdot \Delta_{i}, S \cdot \Delta_{i+1}, S \cdot \Delta_{i+2},\left|F_{i}^{n+1}\right|\right)\right]
$$

\section{1-D Stress Wave Simulation}

1-D stress wave arising from a flyer impacting target is simulated respectively using pure SPH, SPH coupled with artificial viscosity and SPH coupled with FCT. The velocity of flyer is $50 \mathrm{~m} / \mathrm{s}$, the constitutive model of target is ideal elastic(see Table 1).

Table 1 Constitutive Model Parameter of Target

\begin{tabular}{ccc}
\hline$\rho\left(\mathrm{kg} / \mathrm{m}^{3}\right)$ & $K(\mathrm{GPa})$ & $G(\mathrm{GPa})$ \\
\hline 7800 & 222.5 & 85.3
\end{tabular}

Waveforms at 2 different times obtained by pure SPH are seen in Fig. 1, in which the curve at $28 \mu \mathrm{s}$ is wave reflected by free surface. Oscillations behind wave caused by dispersion effect are very obvious in Fig. 1, regardless of loading wave and unloading wave.

Fig. 2 gives waveforms obtained by SPH coupled with artificial viscosity, showing that artificial viscosity can substantially eliminate unphysical oscillations after loading wave, while there are tiny 
oscillations behind the unloading wave. And it makes the curve too smooth to match the theoretical curve.

Fig. 3 shows the waveforms simulated by SPH coupled with FCT. These curves are much sharper than those in Fig. 2, and the tiny oscillations behind unloading wave is eliminated. The results of simulation indicate that the loading/unloading process of strong discontinuity wave can be captured accurately by SPH coupled FCT.
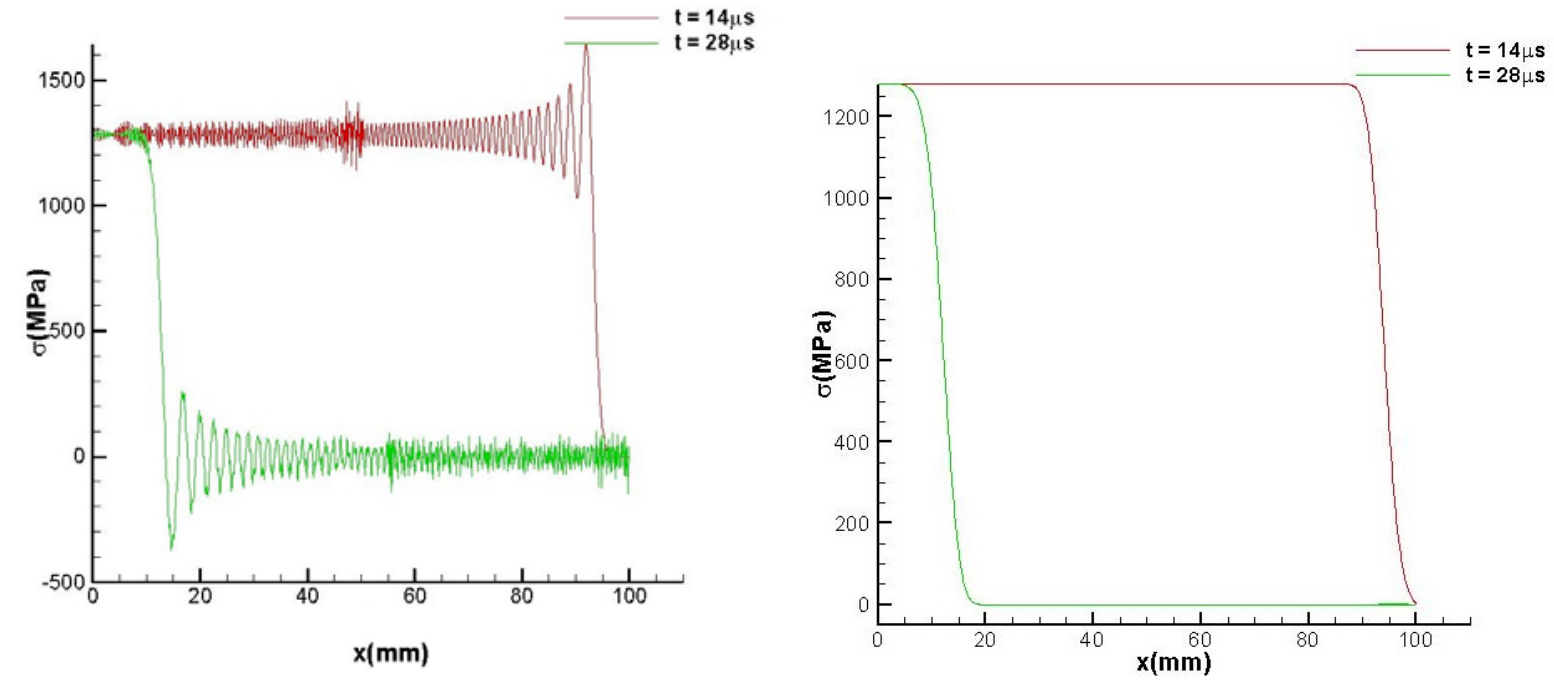

Fig. 1 Waveforms captured by pure SPH

Fig. 2 Waveforms captured by SPH with artificial viscosity

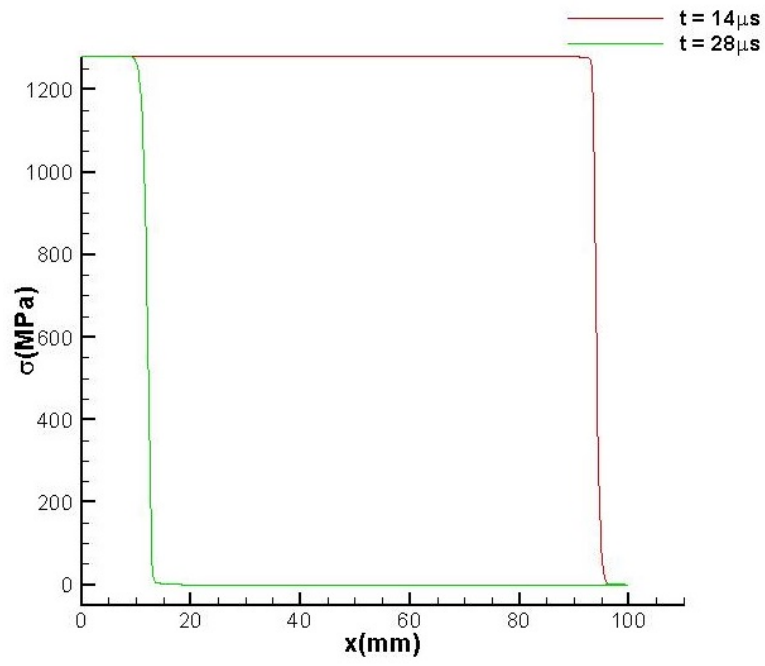

Fig. 3 Waveforms captured by SPH with FCT

\section{Summary}

In this paper, the limit-condition of anti-diffusion from Boris and Book is modified, in order to eliminate oscillations behind wave which are caused by high-order dispersion. A code is developed by embedding the modified FCT method into SPH method. 1-D stress wave is simulated using that code, proving that the new method is effective and accurate in shock wave simulation.

\section{References}

[1] Boris J P and Book D L: Journal of Computational Physics, Vol. 11(1973), No. 1, p. 38.

[2] Book D L, Boris J P and Hain K: Journal of Computational Physics, Vol. 18(1975), No. 3, p. 248.

[3] Boris J P and Book D L: Journal of Computational Physics, Vol. 20(1976), No. 4, p. 397. 
[4] Zalesak S T: Journal of Computational Physics, Vol. 31(1979), No. 3, p. 335.

[5] G. R. Liu, M. B. Liu, Smoothed Particle Hydrodynamics - a Meshfree Particle Method, first ed., World Scientific Publishing Co. Pte. Ltd., River Edge, 2003.

[6] Monaghan J. J.: Journal of Computational Physics, Vol. 82(1989), No. 11, p. 1. 